Leukemia Research

\title{
Screening of a heptamer-type sgRNA library for potential therapeutic agents against hematological malignancies
}

\begin{abstract}
Masayuki Takahashi ${ }^{\text {a }}$, Reyad A. Elbarbary ${ }^{\text {a }}$, Norihiro Watanabe ${ }^{\text {a,b }}$, Atsushi Goto ${ }^{\text {a }}$, Daichi Kamiya ${ }^{a}$, Yoshihiro Watabe ${ }^{a}$, Takayoshi Uchiyama ${ }^{b}$, Miwako Narita ${ }^{b}$, Masuhiro Takahashi ${ }^{b}$, Yoshiaki Takahashi ${ }^{c}$, Noriko Ishihara ${ }^{d}$, Tatsuya Miyazawa ${ }^{e}$, Tetsuo Yoshida ${ }^{\mathrm{e}}$, Mitsuoki Kawano ${ }^{\text {a,f }}$, Masato Tamura ${ }^{\mathrm{f}}$, Masayuki Nashimoto ${ }^{\mathrm{a}, *}$
\end{abstract}

${ }^{\text {a }}$ Department of Applied Life Sciences, Niigata University of Pharmacy and Applied Life Sciences, Higashijima 265-1, Akihaku, Niigata, Niigata 956-8603, Japan

${ }^{\mathrm{b}}$ Laboratory of Hematology and Oncology, Niigata University Graduate School of Health Sciences, Asahimachi-dori 746-2, Chuoku, Niigata, Niigata 951-8518, Japan

${ }^{c}$ Laboratory of Biochemistry, Niigata University Graduate School of Health Sciences,

Asahimachi-dori 746-2, Chuoku, Niigata, Niigata 951-8518, Japan

d Department of Clinical Pathology, Saiseikai Niigata Daini Hospital, Terachi 280-7, Nishiku, Niigata, Niigata 950-1104, Japan

e Biologics Research Laboratories, Kyowa Hakko Kirin Co., Ltd., Asahimachi 3-6-6, Machida, Tokyo 194-8533, Japan

${ }^{\mathrm{f}}$ Department of Biochemistry and Molecular Biology, Hokkaido University Graduate School of Dental Medicine, North 13, West 7, Sapporo, Hokkaido 060-8586, Japan

* Corresponding author. Tel.: +81 25025 5118; fax: +81 250255021 .

E-mail address: mnashimoto@nupals.ac.jp (M. Nashimoto).

(C) 2014. This manuscript version is made available under the Elsevier user license http://www.elsevier.com/open-access/userlicenseł1.0/ 


\section{ABSTRACT}

tRNase- $\mathrm{Z}^{\mathrm{L}}$-utilizing efficacious (TRUE) gene silencing is an RNA-mediated gene expression control technology that has therapeutic potential. This technology is based on the property of tRNase $\mathrm{Z}^{\mathrm{L}}$ that it can cleave any target RNA at any desired site under the direction of an appropriate artificial small guide RNA (sgRNA). To search for novel potential therapeutic sgRNAs for hematological malignancies, we screened a library composed of $156 \mathrm{sgRNAs}$, and found that $20 \mathrm{sgRNAs}$ can efficiently induce apoptosis in leukemia and/or myeloma cells. Furthermore, we demonstrated that 4 of the 20 sgRNAs can reduce growth rates of HL60 cells in mouse xenograft models.

Keywords: leukemia; multiple myeloma; RNA therapy; TRUE gene silencing; tRNase $\mathrm{Z}^{\mathrm{L}}$; sgRNA 


\section{Introduction}

Hematological malignancies such as leukemia and multiple myeloma are, in a considerable number of cases, still fatal diseases. In the United States, people who will be newly diagnosed with leukemia are estimated to be $\sim 50,000$ annually, and $\sim 20,000$ men and women would die of the disease every year (http://www.cancer.gov/cancertopics/types/leukemia; 2014/03/23). It is estimated that 20,000 people will be newly diagnosed with multiple myeloma each year and that $\sim 10,000$ deaths would take place from the disease annually (http://www.cancer.org/cancer/multiplemyeloma/detailedguide/multiple-myeloma-key-s tatistics; 2014/03/23).

Emerging targeted therapy is expected to surpass conventional treatments such as cytotoxic chemotherapy and bone marrow transplantation and to remedy the high fatality of hematological malignancies [1]. A tyrosine-kinase inhibitor, imatinib, which highly selectively targets the BCR-ABL fusion protein that causes chronic myelogenous leukemia (CML), has been shown to be extremely effective in inducing apoptosis of CML cells, and survival of CML patients treated with imatinib has been shown to be not significantly different from that of a general population [2]. A proteasome inhibitor, carfilzomib, which induces apoptosis in myeloma cells, in combination with 
lenalidomide and dexamethasone has been reported to work very effectively on multiple myeloma patients [3]. However, instances of such successful targeted therapies are currently limited, and, even in these cases, emergence of drug-resistant mutations in the course of cancer cell evolution and subsequent relapse of cancer are intrinsic problems.

Various projects for whole cancer genome sequencing have revealed not only common and unique base substitution mutations that contain expected and unexpected ones but also catastrophic chromosomal rearrangements [4-6]. Highly sophisticated bioinformatic analysis of whole cancer genome sequence data has also unveiled genome evolution of each individual cancer [7]. Genuine information extracted from enormous data of cancer genome sequences would help find a large number of new genes, to which cancer cells are addicted or the downregulation of which triggers synthetic lethality. And this would definitely spur development of new targeted therapeutic drugs for hematological malignancies. However, because cancer genomes are intrinsically complex and changeable, so that we cannot easily find effective drugs that eliminate cancer cells [8], truly innovative approaches to find therapeutic agents would be needed. It is well established that hundreds of miRNA species together with various proteinaceous factors such as Argonaute 2 form an elaborate gene modulatory system in human cells, the abnormality of which can cause various types of cancers [9]. 
Pri-miR-106-363 overexpression is observed in a subset of human T-cell leukemias [10], whereas miR-19a/b, miR-21, and miR-181a/b are overexpressed in multiple myeloma cells [11]. Recently we have shown several lines of evidence that cytosolic tRNase $\mathrm{Z}^{\mathrm{L}}$ together with cellular small guide RNAs (sgRNAs) appears to form another broad gene regulatory network $[12,13]$. In human cells, cytosolic $\Delta 30$ tRNase $\mathrm{Z}^{\mathrm{L}}$ interacts with mRNAs to modulate gene expression by cleaving them under the direction of cellular small noncoding RNAs such as 5'-half-tRNA and miRNA as sgRNAs, whereas nuclear full-length tRNase $\mathrm{Z}^{\mathrm{L}}$ works on precursor tRNAs to process them by removing their $3^{\prime}$ trailer. We have shown that the PPM1F and DYNC1H1 mRNAs are directly downregulated by cytosolic tRNase $\mathrm{Z}^{\mathrm{L}}$ guided by a 5'-half-tRNA ${ }^{\mathrm{Glu}}$ and a $28 \mathrm{~S}$ rRNA 3'-terminal fragment, respectively, and that the HDAC4 mRNA is indirectly upregulated by increasing tRNase $\mathrm{Z}^{\mathrm{L}}$ and/or 5'-half-tRNA ${ }^{\mathrm{Glu}}$ levels [12].

Based on this network, we have been developing one of the RNA-mediated gene expression control technologies that have therapeutic potential, called tRNase $\mathrm{Z}^{\mathrm{L}}$-utilizing efficacious gene silencing (TRUE gene silencing) [14]. This technology exploits the property that $\mathrm{tRNase} \mathrm{Z}^{\mathrm{L}}$ can cleave any target RNA at any desired site under the direction of an artificial sgRNA by recognizing a pre-tRNA-like or micro-pre-tRNA-like complex formed between the target RNA and the sgRNA [15-22]. 
The efficacy of TRUE gene silencing has been demonstrated by introducing to living cells various artificially-designed sgRNAs, which can be categorized into four types, 5'-half-tRNA, 14-nt linear RNA, heptamer RNA, and hook RNA [23-28]. Recently, we have shown that sgRNA can be taken up by cells without any transfection reagents and that naked heptamer-type sgRNAs targeting BCL2 and WT1 mRNAs can efficiently induce apoptosis in human leukemia cells [29,30].

These successes encouraged us to search for novel potential therapeutic heptamer-type sgRNAs for hematological malignancies and to identify their targets. Heptamer-type sgRNAs would be advantageous in that they are easier, more accurate and cheaper to synthesize than longer sgRNAs and that cells appear to take up heptamers more easily [31]. We constructed an sgRNA library composed of 156 heptamer-type sgRNAs, and examined how the sgRNAs affect viability of leukemia and myeloma cells. And we found that 20 of the 156 sgRNAs can efficiently induce apoptosis in at least one of the cancer cell lines. Furthermore, we demonstrated that 4 of the 20 effective sgRNAs can reduce tumor growth rates in mouse xenograft models.

\section{Materials and methods}

\subsection{RNA preparation}


Fully 2'-O-methylated, 5'- and 3'-phosphorylated heptamer-type sgRNAs were chemically synthesized with a DNA/RNA synthesizer and subsequently purified through high-performance liquid chromatography with a buffer containing acetonitrile/triethylammonium acetate (TEAA) or acetonitrile/n-hexylammonium acetate (HAA) by Nippon Bioservice (Asaka, Saitama, Japan).

\subsection{Cell culture}

Human cell lines, HL60 [32], KMM-1 [33], RPMI-8226 [34], Oda (established by NI from a 61-year-old Japanese male with multiple myeloma in 1977;

Immuno-Biological Laboratories Co., Gunma, Japan), and K562 [35], were cultured in RPMI-1640 (Wako, Osaka, Japan) supplemented with 10\% fetal bovine serum (MP Biomedicals Japan, Tokyo, Japan) and 1\% penicillin-streptomycin (Invitrogen Japan, Tokyo, Japan) at $37{ }^{\circ} \mathrm{C}$ in $5 \% \mathrm{CO}_{2}$ humidified incubator. And HeLa [36] and HEK293 [37] cells were cultured in DME media (Wako, Osaka, Japan) likewise.

\subsection{Cell viability assay}

The various human cells were plated at $10^{3}$ cells/100 $\mu \mathrm{l} /$ well on a $96-$ well dish in media containing $1 \mu \mathrm{M}$ of various naked heptamer-type sgRNAs. After 3 days, the 
viable cell counts were quantitated with TetraColor ONE (Seikagaku Biobusiness Corp., Tokyo, Japan) [38].

\subsection{Flow cytometry}

HL60 cells were plated at $10^{3}$ cells/100 $\mu \mathrm{l} /$ well on a $96-$ well dish in RPMI-1640 media containing $1 \mu \mathrm{M}$ of various naked heptamer-type sgRNAs. After 3 days, the cells were stained with phycoerythrin (PE)-conjugated Annexin V (BD Pharmingen, San Diego, CA) and 7-AAD (Sigma Chemical Co., St. Louis, MO) at room temperature for 15 min in the dark, and analyzed on a flow cytometer (FACSCalibur).

\subsection{Caspase assay}

HL60 cells were plated at $10^{3}$ cells/well in a 96-well dish in media $(100 \mu \mathrm{l})$ containing $1 \mu \mathrm{M}$ of various naked heptamer-type sgRNAs. After 16 hours, cellular activities of caspases 3/7, 8, and 9 were quantitated with the Caspase-Glo 3/7 Assay system, the Caspase-Glo 8 Assay system, and the Caspase-Glo 9 Assay system, respectively (Promega KK, Tokyo, Japan). 


\subsection{Mouse xenograft experiment}

$2 \times 10^{6}$ of HL60 cells were implanted subcutaneously into a female BALB/c nude mouse (Charles River Laboratories, Yokohama, Japan), and $1 \mathrm{nmol}$ of various heptamer-type sgRNAs dissolved in $10-\mu$ l saline without any carrier reagents were subcutaneously administered directly into the implanted place once a day for 5 or 6 consecutive days starting from the day of the implantation. Although, considering practical use, intravenous systemic administration would be more appropriate, experiments with subcutaneous local administration were carried out in this study primarily due to difficulty in obtaining sufficient amounts of sgRNAs. In each experiment, a cohort consisted of eight mice, and they were sacrificed on the day when the tumor volume (length $\times$ width $\times$ height) reached to $1,500 \mathrm{~mm}^{3}$. The data were subjected to the Kaplan-Meier survival analysis. The mouse xenograft experiments were performed in accordance with the Guidelines on the Care and Use of Laboratory Animals issued by Niigata University of Pharmacy and Applied Life Sciences. The protocol was approved by the Committee on the Ethics of Animal Experiments of Niigata University of Pharmacy and Applied Life Sciences (Permit Number: H21-24). All efforts were made to minimize suffering. 


\subsection{DNA microarray analysis}

HL60 cells were plated at $8 \times 10^{4}$ cells/500 $\mu \mathrm{l} /$ well on a 24 -well dish in RPMI-1640 media containing $1 \mu \mathrm{M}$ of various naked heptamer-type sgRNAs. After 18 hours, total RNA was extracted with ISOGEN (Nippon Gene, Tokyo, Japan) from the cells. Cyanine-3 (Cy3) labeled cRNA was prepared from $0.5 \mu \mathrm{g}$ total RNA using the Quick Amp Labeling Kit, one-color (Agilent Technologies, Tokyo, Japan). $1.65 \mu \mathrm{g}$ of the Cy3-labelled cRNA was fragmented at $60{ }^{\circ} \mathrm{C}$ for 30 minutes, and then hybridized to Agilent Whole Human Genome Oligo Microarrays for 17 hours at $65^{\circ} \mathrm{C}$. After hybridization, microarrays were washed, and then dried immediately by brief centrifugation. Slides were scanned on the Agilent DNA Microarray Scanner (G2565CA) using one color scan setting for $4 \mathrm{x} 44 \mathrm{~K}$ array slides. The scanned images were analyzed with Feature Extraction Software 10.5.1.1 (Agilent Technologies). This microarray analysis was carried out by Takara Bio Inc (Shiga, Japan).

\subsection{Statistical analysis}

In the cell viability assay, differences between control and experimental groups were evaluated by the Student's $t$-test. A difference between control and experimental cohorts in Kaplan-Meier curves was evaluated by the logrank test. 


\subsection{Accession number}

Microarray data were deposited in Gene Expression Omnibus

(http://www.ncbi.nlm.nih.gov/geo), accession GSE49578.

\section{Results}

\subsection{Screening of the sgRNA library for the ability to reduce cancer cell viability}

We constructed an sgRNA library composed of 156 RNA heptamers, which correspond to $\sim 1 \%$ of the total heptamer species number 16,384 (Supplementary Table S1). All the heptamers were chemically synthesized as $2^{\prime}-O$-methyl RNA containing $5^{\prime}-$ and 3'-phosphates. We screened the sgRNA library for the ability to reduce cell viability using a human leukemia cell line, HL60, and three human myeloma cell lines KMM-1, RPMI-8226, and Oda. A viable cell count on at least one cell line after 3 days was decreased by $>80 \%$ and $>90 \%$ by 35 and 20 heptamers, respectively, compared with mock (Supplementary Table S1). Four heptamers reduced cell viability by $>80 \%$ in at least two cell lines and one of them did by $>90 \%$. Although there may be micro sequence motifs common to heptamer sequences of the effective sgRNAs, we have failed to find them so far. 
The effects of the heptamers on the cell viability are exemplified in Fig. 1 with respect to $\mathrm{H} 1885, \mathrm{H} 3277, \mathrm{H} 10927$, and $\mathrm{H} 13260$, which reduced viability of HL60 and RPMI- 8226 cells by $>88 \%$ and $>76 \%$, respectively. We performed flow cytometric analysis with annexin V and 7AAD double staining for the HL60 cells treated with these heptamers. In each case, a total cell number (56-95\%) in early and late apoptotic stages was much higher than that (4-6\%) in mock (Fig. 2). We also measured activities of caspases 3/7, 8, and 9 in the HL60 cells treated with those heptamers, and observed that the activities are significantly elevated compared with those in mock (Fig. 3). Together, these results suggest that the reduction in HL60 cell numbers is due to induction of apoptosis. Furthermore, we showed that, in contrast, these effective sgRNAs hardly affect viability of human peripheral blood mononuclear cells (Supplementary Fig. S1).

A subset of the library was also screened for the ability to reduce viability of other human cell lines, K562, HeLa, and non-cancerous HEK293 cells. The tested heptamers hardly affect HEK293 cell viability in most cases, and reduced viability of K562 and HeLa cells by $0-21 \%$ and $0-11 \%$, respectively (Supplementary Table S1). 
Furthermore, we carried out mouse xenograft experiments to examine how the highly efficacious heptamer-type sgRNAs H1885, H3277, H10927, and H13260 can reduce the growth of HL60 cells in vivo. HL60 cells were implanted subcutaneously into nude mice, and each sgRNA was subsequently administered locally once a day for 5 or 6 consecutive days. In two independent experiments for each sgRNA, median survival periods of the effective sgRNA cohorts in the Kaplan-Meier curves were greater than those of the control sgRNA cohorts by $2-13$ days, although the data in two experiments were not statistically significant (Fig. 4). These observations suggest that these sgRNAs work in vivo, albeit moderately.

\subsection{DNA microarray analysis for changes in an mRNA profile}

We performed DNA microarray analysis for changes in an mRNA profile in order to find target mRNAs of those effective sgRNAs and to elucidate mechanisms of apoptosis induction. Gene expression profiles in HL60 cells were analyzed after 18-hour culture in the presence of the four effective sgRNAs and the control sgRNA H5882 by comparing them with a profile in untreated HL60 cells. To search for potential target mRNAs specific to each effective sgRNA, we listed genes, the expression of which was reduced by more than $34 \%$ only in the presence of the 
corresponding sgRNA. And two to five candidate genes were found for the four

effective heptamer-type sgRNAs (Fig. 5A). Furthermore, we looked for potential target sites in mRNA from each gene and found at least one mRNA that contains a potential tRNase $Z^{\mathrm{L}}$ target site for each sgRNA (Fig. 5B).

In addition to these specific mRNAs, a subset of the other mRNAs, the levels of which were downregulated by more than $34 \%$ in more than one samples of HL60 cells treated with the sgRNAs, can be targets of the sgRNAs. We also cannot exclude the possibility that long noncoding RNAs and/or pri-miRNAs are their genuine targets because the DNA microarray that we used included only a limited number of probes for such noncoding RNAs.

\section{Discussion}

By screening the heptamer-type sgRNA library containing 156 heptamers, we found 20 sequences that are highly effective in inducing apoptosis of human leukemia and/or myeloma cells. We would be able to find $~ 2,000$ highly effective heptamers in the full sgRNA library with 16,384 heptamers, if the probability to find effective heptamers is nearly constant. Genome abnormality in each cancer patient differs and cancer cells evolve generating drug-resistant mutations in time lapse. Thus, as many 
effective heptamers as possible should be stored for literally "tailor-made" treatments for hematological malignancies. Information extracted from whole cancer genome sequencing data would be expected to help identify therapeutic target genes, to which cancer cells are addicted or the downregulation of which trigger synthetic lethality. Considering unusual complexity of human cells [8], however, screening a large potential therapeutic agent library would be currently superior to testing drugs to target a specific gene one by one. The collection of $\sim 2,000$ potential therapeutic sgRNAs may be sufficient to fulfill tailor-made treatments for hematological malignancies.

Alternatively we may need to extend the library to one that consists of a full set of $\sim 2.7$ $\times 10^{8}$ different heptamer pairs, the screening of which would give us a chance to find sgRNA cocktails that work synergistically as mentioned below.

We showed that 39, 7, and 5 heptamer-type sgRNAs reduce viability of HL60, KMM-1, and RPMI-8226 cells, respectively, by $>70 \%$, whereas tested sgRNAs reduced viability of Oda, K562, HeLa, and HEK293 cells only by $0-57 \%, 0-21 \%, 0-11 \%$, and 0-21\%, respectively (Supplementary Table S1). These differences in susceptibility to sgRNA among the cell lines may be attributed to differences in sgRNA biostability, subcellular localization of taken-up sgRNA, and/or the cellular tRNase $\mathrm{Z}^{\mathrm{L}}$ level. It should be noted that the relative degree of the viability-reducing effect of sgRNA 
among the cell lines differs depending on each sgRNA. This suggests that each sgRNA has its own anti-apoptotic target(s), which affect cell survivability differentially depending on cell lines.

Although the DNA microarray data suggested target mRNA(s) for each sgRNA (Fig. 5), the downregulation of which may trigger synthetic lethality, further experiments are needed to elucidate molecular mechanisms of apoptosis induction. The sgRNAs may also target pri-miRNAs and/or long noncoding RNAs to induce apoptosis [9,38]. Alternatively, it would be possible that the heptamers interact with proteins to interfere with their functions or bind to DNA sequences to affect nearby gene expression.

Heptamer-type sgRNAs synthesized with a DNA/RNA synthesizer were purified through high-performance liquid chromatography with a buffer containing acetonitrile/TEAA or acetonitrile/HAA. In the course of this study, we noticed that synthetic heptamers eluted with the HAA buffer appeared to be more active in inducing apoptosis of leukemia and myeloma cells than those eluted with the TEAA buffer (data not shown). This may be because cellular uptake and/or vesicle-to-cytosol release of the sgRNAs with hexylammonium cations as counter ions might be more efficient. We may 
be able to improve the efficacy of heptamer-type sgRNA by elucidating how counter cations affect the sgRNA's efficacy and selecting better counter cations.

We also examined if heptamer-type sgRNA cocktails work additively or synergistically, using several pairs of heptamers, each of which shows a relatively weak activity. Although, in most cases, heptamer cocktails showed only an additive effect, a cocktail of H13693 and H15785 worked synergistically in reducing viability of RPMI-8226 cells (Supplementary Fig. S2A). This suggests that products of each heptamer's target mRNAs may work cooperatively in the cells. Alternatively, this cocktail happened to work like a double heptamer, which can bind an mRNA molecule consecutively to function as a 14-nt linear sgRNA by chance [27]. In fact, the TRIB3 and PIKFYVE mRNAs contain a sequence complementary to the 14-nt sgRNA (Supplementary Fig. S2B), and they may have been additional targets of the double heptamer.

Synergistic effect was also observed in a mouse xenograft experiment, where a cocktail of three different heptamers reduced HL60 cell growth drastically, although the cocktail did not show the synergistic effect in vitro (data not shown). The synergistic effect in vivo may be attributed to an effect on the tumor microenvironment through targeting unknown mRNAs in host stromal cells. 
Although this and previous studies $[29,30]$ suggest that heptamer-type sgRNAs based on TRUE gene silencing might become therapeutic agents against hematological malignancies, animal experiments for systemic administration effect, toxicity, and pharmacokinetics, and elucidation of mechanisms of apoptosis induction are needed before clinical trials. In spite of the apparent disadvantages, 5'-half-tRNA-type or linear-type sgRNAs would be worth being further investigated for their potential as therapeutic agents. And final forms of tailor-made therapeutic sgRNAs for hematological malignancies may be in combination with other targeted drugs made of small molecules or antibodies.

\section{Conflict of interest statement}

The authors TM and TY are employees of Kyowa Hakko Kirin. Niigata University of Pharmacy and Applied Life Sciences and Niigata University have a pending patent application on a subset of the heptamer-type sgRNAs. The authors MNarita, MasuhiroT TM, TY and MNashimoto are named as inventors in this application.

\section{Acknowledgements}


We thank Miharu Takeda for technical assistance.

Contributions. MasayukiT designed and performed the experiments, analyzed the data, and wrote the paper; RAE, NW, AG, DK, YW, TU, MNarita, MasuhiroT, and MTamura performed the experiments and analyzed the data; YT NI TM TY MK analyzed the data; MNashimoto designed the experiments, analyzed the data, and wrote the paper.

Role of the funding source. This work was supported by Adaptable and Seamless Technology Transfer Program through Target-driven R\&D, Japan Science and Technology Agency, the Science Research Promotion Fund from the Promotion and Mutual Aid Corporation for Private Schools of Japan, and the JSPS KAKENHI Grant Numbers 24300342 and 11J08925. The funders had no role in study design, data collection and analysis, decision to publish, or preparation of the manuscript. 


\section{References}

[1] Sawyers C. Targeted cancer therapy. Nature 2004;432:294-7.

[2] Gambacorti-Passerini C, Antolini L, Mahon FX, Guilhot F, Deininger M, Fava C, et al. Multicenter independent assessment of outcomes in chronic myeloid leukemia patients treated with imatinib. J Natl Cancer Inst 2011;103:553-61.

[3] Jakubowiak AJ, Dytfeld D, Griffith KA, Lebovic D, Vesole DH, Jagannath S, et al. phase $1 / 2$ study of carfilzomib in combination with lenalidomide and low-dose dexamethasone as a frontline treatment for multiple myeloma. Blood 2012;120:1801-9.

[4] Chapman MA, Lawrence MS, Keats JJ, Cibulskis K, Sougnez C, Schinzel AC, et al. Initial genome sequencing and analysis of multiple myeloma. Nature 2011; 471:467-72.

[5] Stephens PJ, Greenman CD, Fu B, Yang F, Bignell GR, Mudie LJ, et al. Massive genomic rearrangement acquired in a single catastrophic event during cancer development. Cell 2011;144:27-40.

[6] Magrangeas F, Avet-Loiseau H, Munshi NC, Minvielle S. Chromothripsis identifies a rare and aggressive entity among newly diagnosed multiple myeloma patients. Blood 2011;118:675-8. 
[7] Egan JB, Shi CX, Tembe W, Christoforides A, Kurdoglu A, Sinari S, et al.

Whole-genome sequencing of multiple myeloma from diagnosis to plasma cell leukemia reveals genomic initiating events, evolution, and clonal tides. Blood 2012;120:1060-6.

[8] Koch C. Modular Biological Complexity. Science 2012;337:531-2.

[9] Yates LA, Norbury CJ, Gilbert RJ. The long and short of microRNA. Cell 2013;153:516-9.

[10] Landais S, Landry S, Legault P, Rassart E. Oncogenic potential of the miR-106-363 cluster and its implication in human T-cell leukemia. Cancer Res 2007;67:5699-707.

[11] Pichiorri F, Suh SS, Ladetto M, Kuehl M, Palumbo T, Drandi D, et al. MicroRNAs regulate critical genes associated with multiple myeloma pathogenesis. PNAS 2008;105:12885-90.

[12] Elbarbary RA, Takaku H, Uchiumi N, Tamiya H, Abe M, Takahashi M, et al. Modulation of gene expression by human cytosolic tRNase $\mathrm{Z}^{\mathrm{L}}$ through 5'-half-tRNA, PLoS ONE 2009;4:e5908.

[13] Elbarbary RA, Takaku H, Uchiumi N, Tamiya H, Abe M, Nishida H, et al. Human cytosolic tRNase $\mathrm{Z}^{\mathrm{L}}$ can downregulate gene expression through miRNA. FEBS Lett 
2009;583:3241-6.

[14] Scherer L, Rossi JJ. Therapeutic potential of RNA-mediated control of gene expression: Options and designs. In: Morris KV, editor. RNA and the Regulation of Gene Expression: A hidden layer of complexity, Norfolk: Caister Academic Press Inc; 2008, p. 201-26.

[15] Nashimoto M. Conversion of mammalian tRNA 3' processing endoribonuclease to four-base-recognizing RNA cutters. Nucleic Acids Res 1995;23:3642-7.

[16] Nashimoto M. Specific cleavage of target RNAs from HIV-1 with 5' half tRNA by mammalian tRNA 3' processing endoribonuclease. RNA 1996;2:2523-4.

[17] Nashimoto M, Geary S, Tamura M, Kasper R. RNA heptamers that directs RNA cleavage by mammalian tRNA 3' processing endoribonuclease. Nucleic Acids Res 1998;26:2565-71.

[18] Nashimoto M. Anomalous RNA substrates for mammalian tRNA 3' processing endoribonuclease. FEBS Lett 2000;472:179-86.

[19] Takaku H, Minagawa A, Masamichi T, Nashimoto M. A candidate prostate cancer susceptibility gene encodes tRNA 3' processing endoriobonuclease. Nucleic Acids Res $2003 ; 31: 2272-8$.

[20] Takaku H, Minagawa A, Takagi M, Nashimoto M. The N-terminal half-domain of 
the long form of tRNase $\mathrm{Z}$ is required for the RNase 65 activity. Nucleic Acids Res 2004;32:4429-38.

[21] Takaku H, Minagawa A, Takagi M, Nashimoto M. A novel four-base-recognizing RNA cutter that can remove the single 3' terminal nucleotides from RNA molecules. Nucleic Acids Res 2004;32:e91.

[22] Shibata HS, Takaku H, Takagi M, Nashimoto M. The T loop structure is dispensable for substrate recognition by tRNase ZL. J Biol Chem $2005 ; 280: 22326-34$

[23] Tamura M, Nashimoto C, Miyake N, Daikuhara Y, Ochi K, Nashimoto M. Intracellular mRNA cleavage by $3^{\prime}$ tRNase under the direction of $2^{\prime}-O$-methyl RNA heptamers. Nucleic Acids Res 2003;31:4354-60.

[24] Habu Y, Miyano-Kurosaki N, Kitano M, Endo Y, Yukita M, Ohira S, et al. Inhibition of HIV-1 gene expression by retroviral vector-mediated small-guide RNAs that direct specific RNA cleavage by tRNase ZL. Nucleic Acids Res $2005 ; 33: 235-43$.

[25] Nakashima A, Takaku H, Shibata HS, Negishi Y, Takagi M, Tamura M, et al. Gene-silencing by the tRNA maturase tRNase $\mathrm{Z}^{\mathrm{L}}$ under the direction of small guide RNA. Gene Ther 2007;14:78-85. 
[26] Elbarbary RA, Takaku H, Tamura M, Nashimoto M. Inhibition of vascular endothelial growth factor expression by TRUE gene silencing. Biochem and Biophys Res Commun 2009;379:924-7.

[27] Sano T, Takahashi M, Nozaki T, Takahashi Y, Tamura M, Nashimoto M. Expanding the utility of heptamer-type sgRNA for TRUE gene silencing. Biochem and Biophys Res Commun 2011;416:427-32.

[28] Takahashi M, Elbarbary RA, Abe M, Sato M, Yoshida T, Yamada Y, et al. Elimination of specific miRNAs by naked 14-nt sgRNAs. PLoS ONE 2012;7:e38496.

[29] Takahashi M, Elbarbary RA, Nakashima A, Abe M, Watanabe N, Narita M, et al. A naked RNA heptamer targeting the human Bcl-2 mRNA induces apoptosis of HL60 leukemia cells. Cancer Lett 2013;328:362-8.

[30] Watanabe N, Narita M, Yamahira A, Taniguchi T, Furukawa T, Yoshida T, et al. Induction of apoptosis of leukemic cells by TRUE gene silencing using small guide RNAs targeting the WT1 mRNA. Leukemia Res 2013;37:580-5.

[31] Loke SL, Stein CA, Zhang XH, Mori K, Nakanishi M, Subasinghe C, et al. Characterization of oligonucleotide transport into living cells. PNAS 1989;86:3474-8. 
[32] Gallagher R, Collins S, Trujillo J, McCredie K, Ahearn M, Tsai S, et al.

Characterization of the continuous, differentiating myeloid cell line (HL-60) from a patient with acute promyelocytic leukemia. Blood 1979;54:713-33.

[33] Togawa A, Inoue N, Miyamoto K, Hyodo H, Namba M. Establishment and characterization of a human myeloma cell line (KMM-1). Int J Cancer 1982;29:495-500.

[34] Matsuoka Y, Moore GE, Yagi Y, Pressman D. Production of free light chains of immunoglobulin by a hematopoietic cell line derived from a patient with multiple myeloma. Proc Soc Exp Biol Med 1967;125:1246-50.

[35] Lozzio CB, Lozzio BB. Human chronic myelogenous leukemia cell-line with positive Philadelphia chromosome. Blood 1975;45:321-34.

[36] Scherer WF, Syverton JT, Gey GO. Studies on the propagation in vitro of poliomyelitis viruses. IV. Viral multiplication in a stable strain of human malignant epithelial cells (strain HeLa) derived from an epidermoid carcinoma of the cervix. J Exp Med 1953;97:695-710.

[37] Graham FL, Smiley J, Russell WC, Nairn R. Characteristics of a human cell line transformed by DNA from human adenovirus type 5. J Gen Virol 1977;36:59-74.

[38] Nakano K, Yamaoka K, Hanami K, Saito K, Sasaguri Y, Yanagihara N, et al. 
Dopamine induces IL-6-dependent IL-17 production via D1-like receptor on CD4 naive T cells and D1-like receptor antagonist SCH-23390 inhibits cartilage destruction in a human rheumatoid arthritis/SCID mouse chimera model. J Immunol 2011;186:3745-52.

[39] Cheetham SW, Gruhl F, Mattick JS, Dinger ME. Long noncoding RNAs and the genetics of cancer. Br J Cancer 2013;108:2419-25. 


\section{Figure Legends}

Fig. 1. Cell viability assays. Relative viable cell counts were measured 72 hours after HL60 cells (A) or RPMI-8226 cells (B) were cultured in the absence or presence of 1 $\mu \mathrm{M}$ of the naked heptamer-type sgRNA H1885, H3277, H10927, H13260, H10944, or H12287. The relative viable cell counts in the absence of sgRNAs are adjusted to 100. *, $\mathrm{P}<0.002$

Fig. 2. Flow cytometry. Flow cytometric analysis for HL60 cells was performed with annexin $\mathrm{V}$ and 7AAD double staining. The cells were analyzed 72 hours after cultured in the absence or presence of $1 \mu \mathrm{M}$ of the naked heptamer-type sgRNA H1885 (A), H3277 (A), H13260 (A), or H10927 (B).

Fig. 3. Caspase assays. Cellular activities of caspases 3/7 (A), 8 (B), and 9 (C) were measured 16 hours after HL60 cells $\left(10^{3}\right.$ cells/100 $\left.\mu 1\right)$ were cultured in the absence or presence of $1 \mu \mathrm{M}$ of the naked heptamer-type sgRNA H1885, H3277, H13260, H10927, or H5882. Error bars indicate SD $(\mathrm{n}=3) .{ }^{*}, \mathrm{P}<0.01$. 
Fig. 4. Kaplan-Meier survival analysis. HL60 cells were implanted subcutaneously into nude mice, and $1 \mathrm{nmol}$ of the effective naked heptamer-type sgRNA H1885 (A), H3277 (B), H10927 (C), or H13260 (D) was administered into the implanted place once a day for 5 or 6 consecutive days. The heptamer-type sgRNA H5882 (Supplementary Table S1) was used as a control. They were sacrificed on the day when the tumor volume reached to $1,500 \mathrm{~mm}^{3}$.

Fig. 5. DNA microarray analysis. (A) Heat maps of DNA microarray data that represent specifically downregulated genes in HL60 cells after 18-hour culture in the presence of the naked heptamer-type sgRNA H1885, H3277, H10927, H13260, or H5882. Genes, the expression of which was greater than 100 in Base gScale Signal and reduced by more than $34 \%$ only in the presence of one of the sgRNAs, were selected. (B) Candidate target mRNAs and potential sgRNA binding sites. Potential tRNase $\mathrm{Z}^{\mathrm{L}}$ target sites in mRNA sequences from the selected genes are shown with bound heptamer-type sgRNAs. The potential target site is defined as a site where there is a sequence complementary to at least six consecutive nucleotides of a heptamer-type sgRNA preceded by a potential hairpin structure containing an at least three-base-pair stem. Potential stem-forming sequences and cleavage site are indicated by underscores and an 
arrow with a number (from the GenBank data), respectively. 

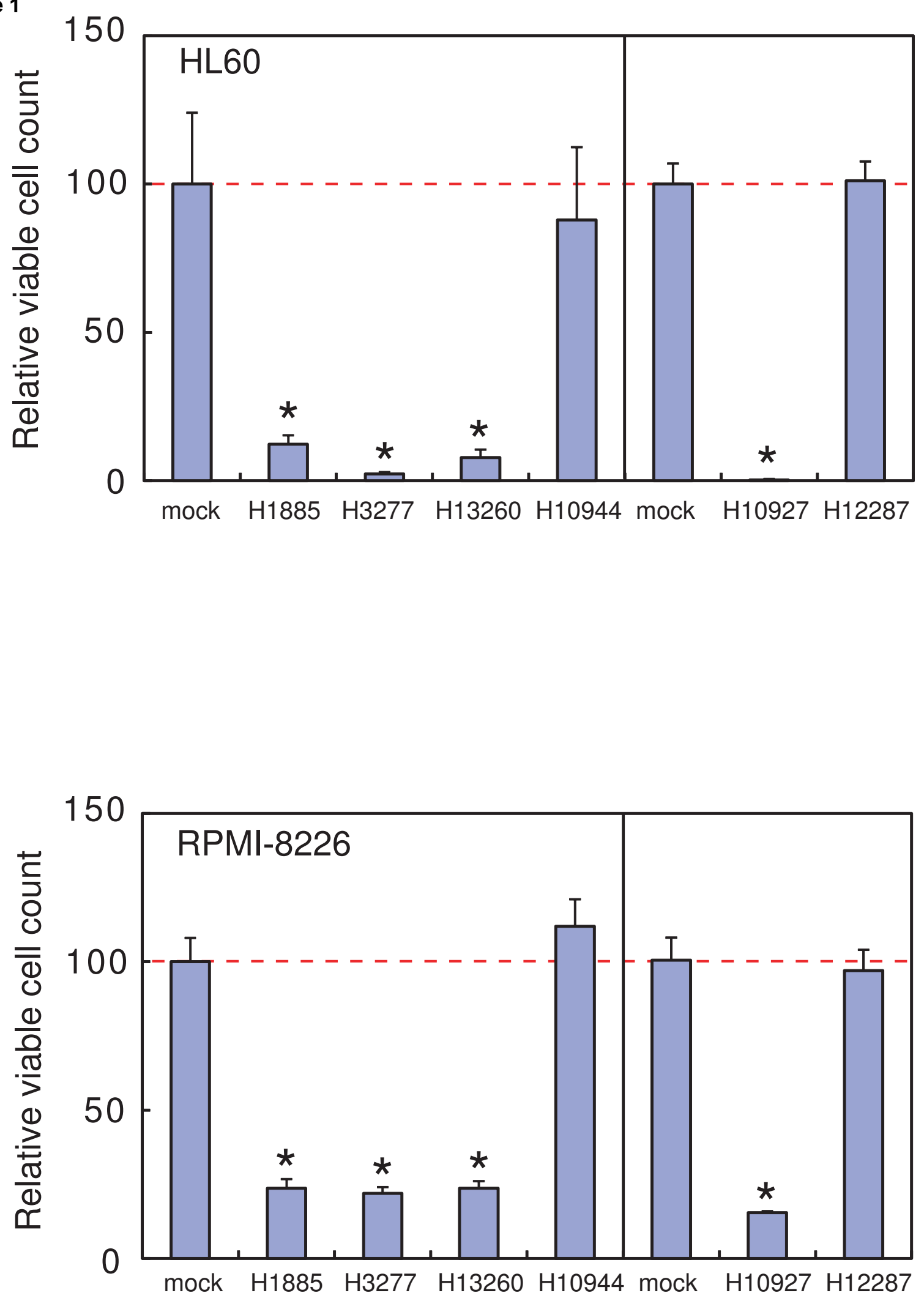

Figure 1 

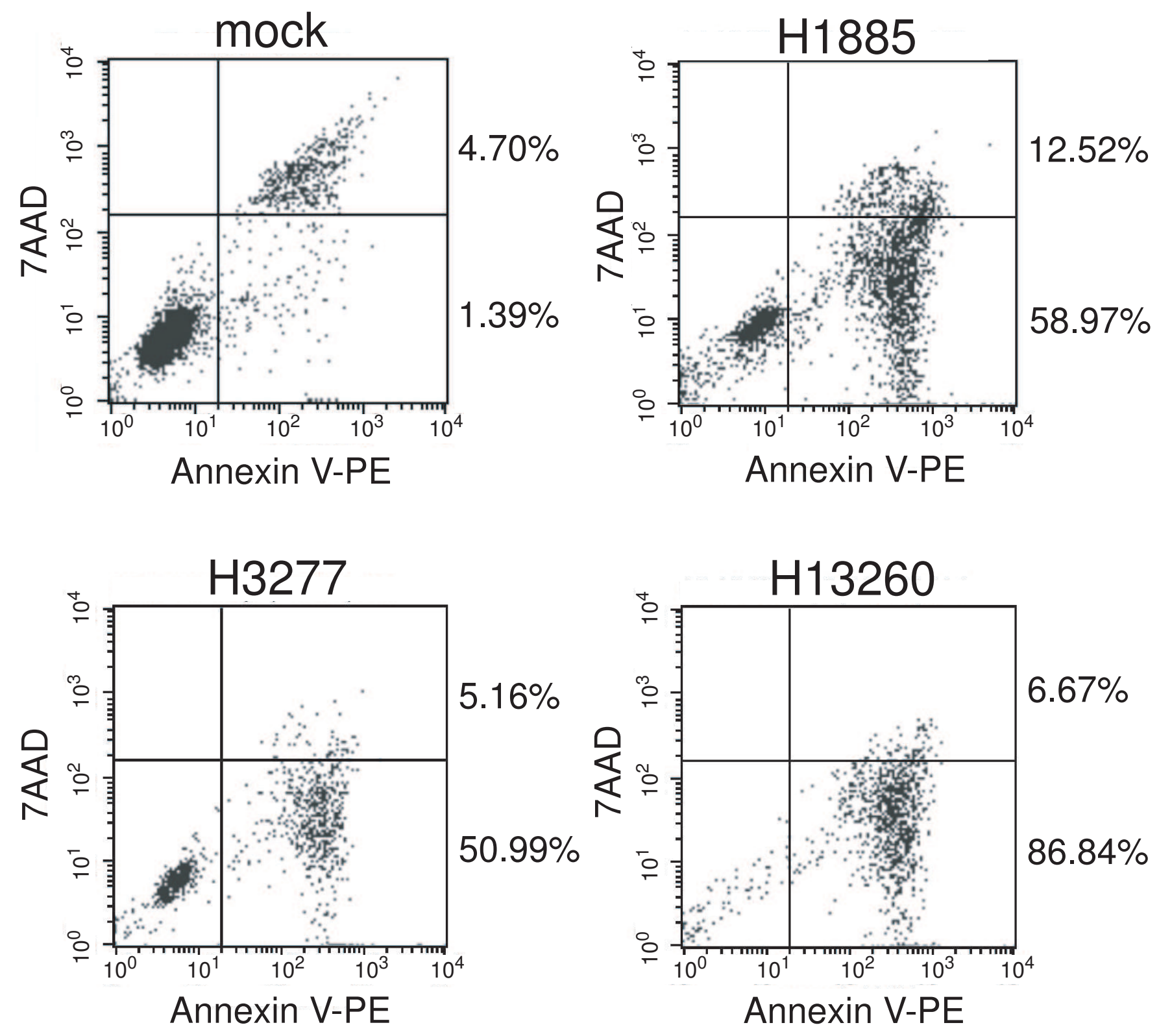

$B$
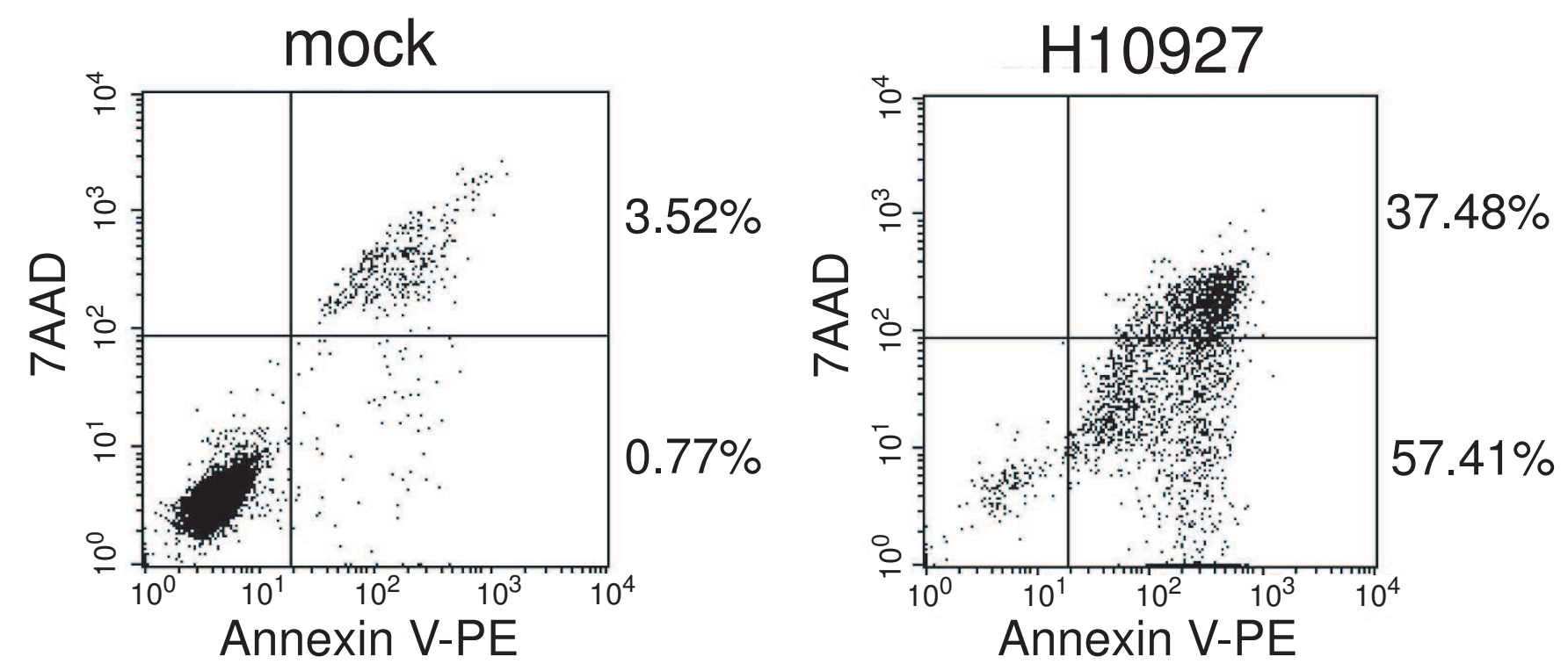

Figure 2 
Aigure 3
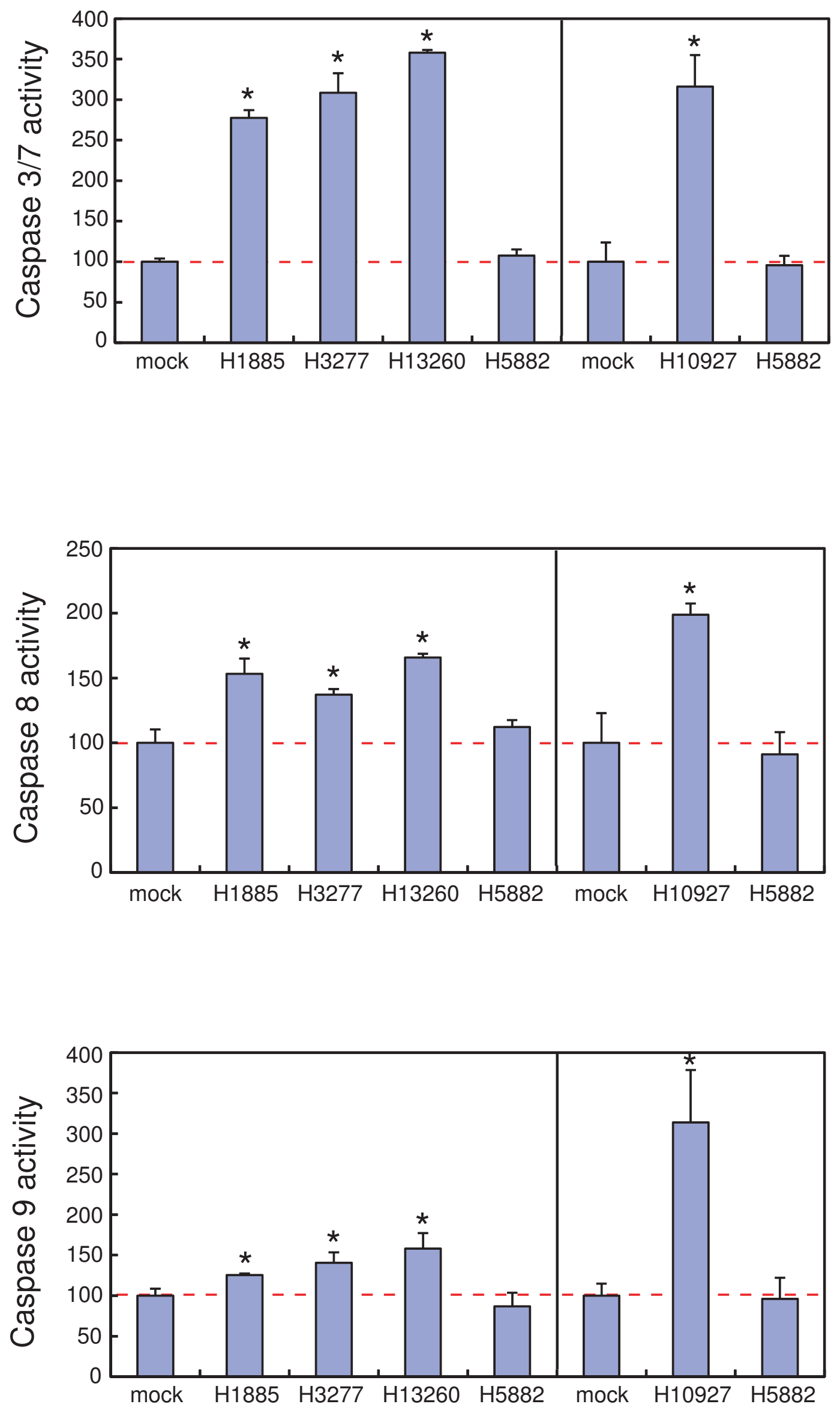

Figure 3 

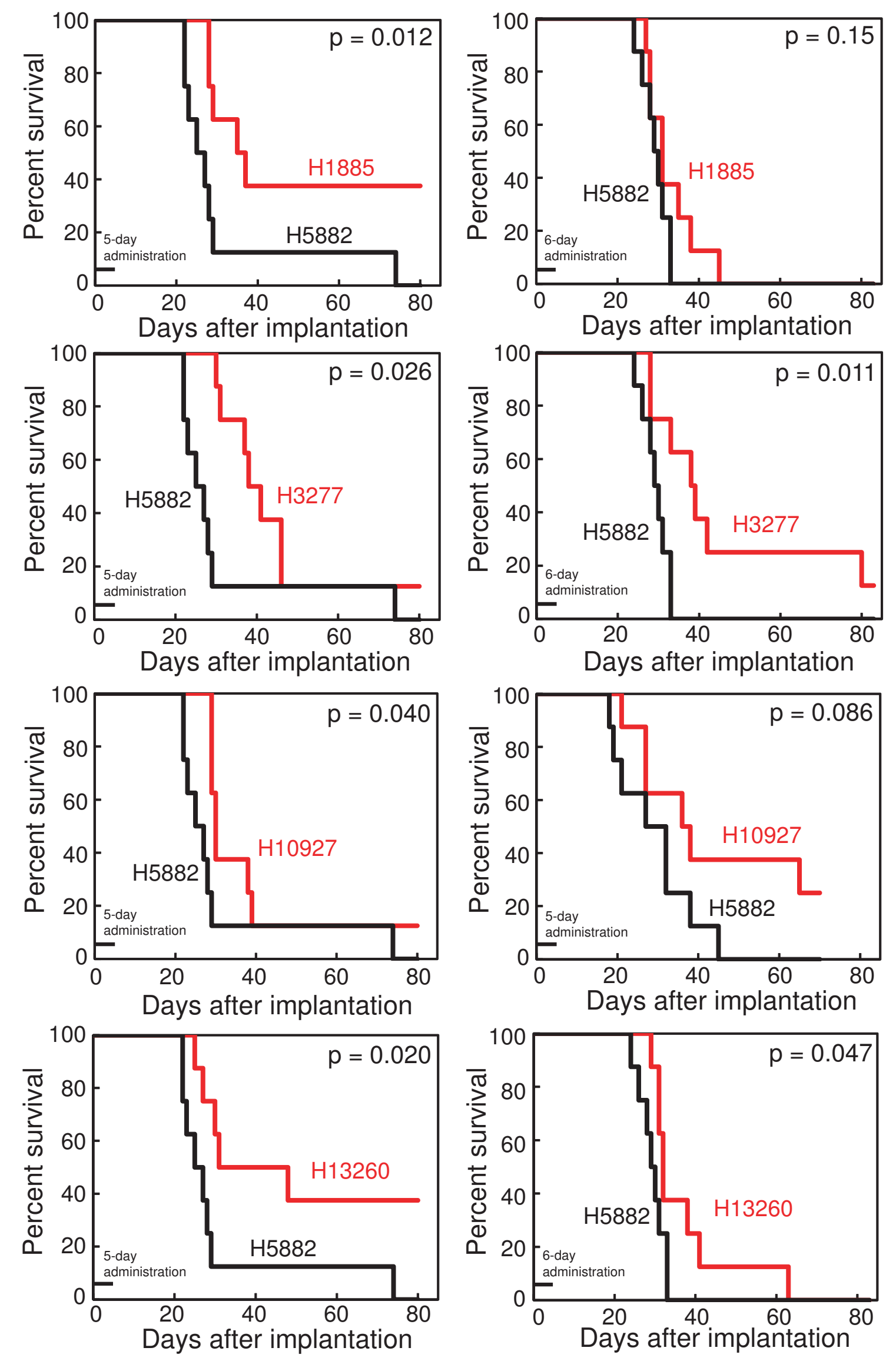

Figure 4 
H1885

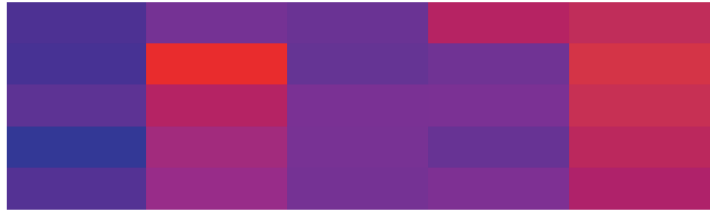

EVK1 (NM_001989.3)

KIAA1751 (NM_001080484.1)

LGI4 (NM_139284.2)

FLJ35379 (AK092698.1)

TMCC2 (NM_014858.3)

H3277

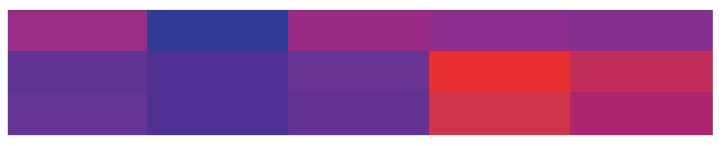

NCR3 (NM_147130.2)

GPHB5 (NM_145171.3)

FBLIM1 (NM_017556.2)

H10927

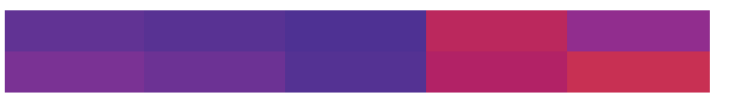

CHRNA4 (X89741.1)

CACNA1E (NM_000721.3)

$\mathrm{H} 13260$

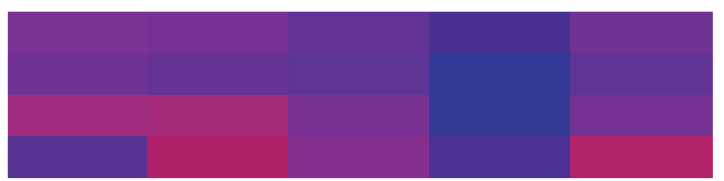

UBE2E1 (NM_003341.4)

PPP3R1 (NM_000945.3)

MED18 (NM_017638.2)

JAKMIP3 (AL832756.1)

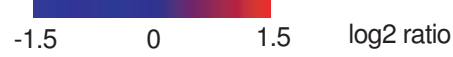

B

H1885

$$
\begin{aligned}
& 3^{\prime} \text { ACUUCUA }{ }^{\prime \prime}
\end{aligned}
$$

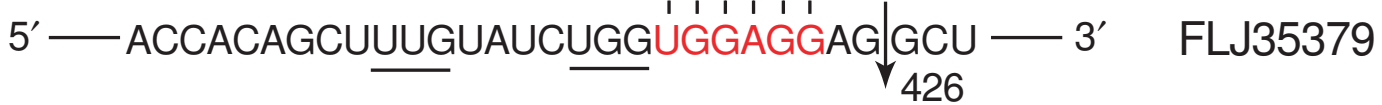

H3277

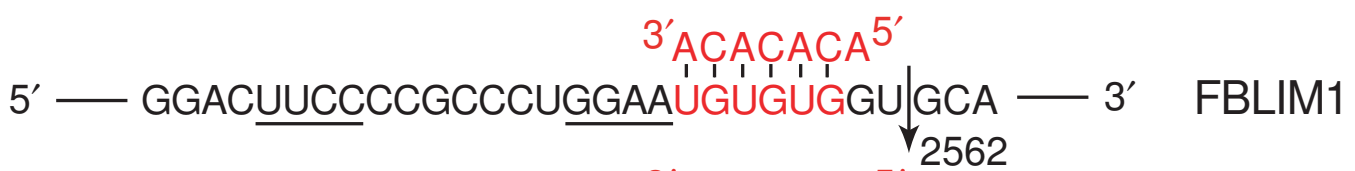

$3^{\prime} A_{1} A_{1} C_{1} A_{1} A^{5}$

5' — UGUGGUCCUUGGCAAGUGAU'GU'GU'GAG|UCC — $3^{\prime}$ NCR3

H10927

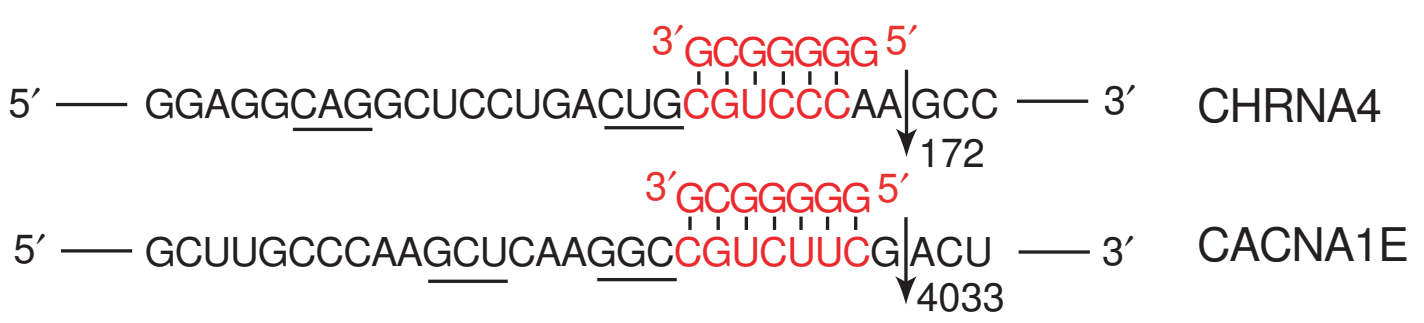

H13260

$$
\begin{aligned}
& 3^{\prime} \text { CGACCAC 5' } \\
& \text { 5' - AGAAAUGGGGAUUCACCAUG'G'U'G'G'GU'CA|GGC — 3' MED18 }
\end{aligned}
$$

Figure 5 